\title{
A Robust Energy Saving Data Dissemination Protocol for IoT-WSNs
}

\author{
Moonseong Kim ${ }^{1}$, Sooyeon Park ${ }^{2}$ and Woochan Lee ${ }^{2}$ \\ ${ }^{1}$ Department of Liberal Arts, Seoul Theological University, Bucheon, Republic of Korea \\ [e-mail: moonseong@stu.ac.kr] \\ ${ }^{2}$ Department of Electrical Engineering, Incheon National University, Incheon, Republic of Korea \\ [e-mails: \{anisoo, wlee\}@inu.ac.kr] \\ *Corresponding author: Woochan Lee
}

Received May 7, 2018; revised July 2, 2018; accepted August 12, 2018; published December 31, 2018

\begin{abstract}
In Wireless Sensor Networks (WSNs) for Internet of Things (IoT) environment, fault tolerance is a most fundamental issue due to strict energy constraint of sensor node. In this paper, a robust energy saving data dissemination protocol for IoT-WSNs is proposed. Minimized energy consumption and dissemination delay time based on signal strength play an important role in our scheme. The representative dissemination protocol SPIN (Sensor Protocols for Information via Negotiation) overcomes overlapped data problem of the classical Flooding scheme. However, SPIN never considers distance between nodes, thus the issue of dissemination energy consumption is becoming more important problem. In order to minimize the energy consumption, the shortest path between sensors should be considered to disseminate the data through the entire IoT-WSNs. SPMS (Shortest Path Mined SPIN) scheme creates routing tables using Bellman Ford method and forwards data through a multi-hop manner to optimize power consumption and delay time. Due to these properties, it is very hard to avoid heavy traffic when routing information is updated. Additionally, a node failure of SPMS would be caused by frequently using some sensors on the shortest path, thus network lifetime might be shortened quickly. In contrast, our scheme is resilient to these failures because it employs energy aware concept. The dissemination delay time of the proposed protocol without a routing table is similar to that of shortest path-based SPMS. In addition, our protocol does not require routing table, which needs a lot of control packets, thus it prevents excessive control message generation. Finally, the proposed scheme outperforms previous schemes in terms of data transmission success ratio, therefore our protocol could be appropriate for IoT-WSNs environment.
\end{abstract}

Keywords: Internet of Things, Wireless Sensor Networks, Dissemination Protocol, Energy Efficient Data Distribution, Fast Computation, Fault Tolerance 


\section{Introduction}

Internet of Things (IoT) has begun to emerge for nearly a decade and is widely defined as a network connecting things with things based on sensory, communication, networking, and information processing technologies [1][2]. The scope of things involved in IoT includes well-known systems such as RFID, WSN, USN, M2M, and CPS [3][4]. The purpose of connecting IoT devices is to serve as a backbone for enabling smart environments, disaster managements, and military operations to retrieve information [5][6]. For instance, military battlefields in inaccessible or hostile territory could constitute a potential application of WSNs, where WSNs may be necessary to detect enemies and to track their activities [7]. Since these sensor devices operate under strict energy constraints, they have to avoid unnecessary energy consumption as much as possible. Furthermore, the sensor nodes are deployed in uncontrolled and uneven hostile environment. Thus, it is difficult to apply fault tolerance schemes in WSNs [8].

Initially, sensor nodes could be fully connected to transmit sensed data in WSNs. A sensor node permits to retransmit the received information to neighbors or to access it to perform further processing. If partial sensor nodes reach their critical energy level, the network lifetime will decrease. In the worst-case scenario, a sensor fault might lead to unexpected network disconnection, therefore low power consumption and fault tolerance routing techniques for IoT-WSNs are important issues and the problems have been extensively studied in the past few decades [9][10][11].

WSN routing protocols, generally categorized as cluster-based [12] and flat types, could take energy into consideration as an important measure in the path-construction step to prolong WSN lifetime [13]. Cluster-based routing protocols divide the given topology into clusters, and a chosen node (called a cluster head) could manage and control its cluster. Cluster selection schemes have been studied to improve energy-effectiveness, or to achieve uniform power consumption [14][15]. Here, the protocols could consider single or multi-paths in data dissemination step. Single-path routing protocols not only find the shortest path but also choose the following hop based on its remained energy level. By choosing sensor nodes considering larger stored energy during path construction, the protocol could uniformly enforce power consumption [16]. Multipath routing protocols have been used to achieve load balancing. They also strengthen topology stability through multi-paths, which enable the topology to be recovered quickly, whereas in single-path protocols, whenever energy of a sensor is exhausted, a replaced path should be built [17][18].

There are two classical flat types; namely, Flooding scheme and SPIN scheme [19]. First, in Flooding type, any sensor disseminates the received data from all neighbors. It is uncomplicated and rudimentary method. In this scheme, sensors quickly send the sensed information without considering energy consumption, thus they heavily drain batteries if sensed data-storm happens. Second, SPIN resolves the problem by negotiating with neighbors [20]. Even though SPIN interchanges a meta-data, however, to avoid overlapped data, it never considers energy status of nodes and distance to neighbors. It is a burden of sending the received data without exploiting the distance to neighbors to tune the energy of transmission [21].

Since the energy consumption strongly depends on the range between sensors [22], SPMS [23][24] could reduce the energy consumption using multi-hop path based on the shortest path. SPMS lessens the consumed power and shortens disseminated time compared with SPIN, and 
it is fault tolerant. However, some sensors on the shortest path could be frequently accessed and worked, thus network lifetime might be shortened quickly. Furthermore, it has a drawback of maintaining a routing table in a zone of each node.

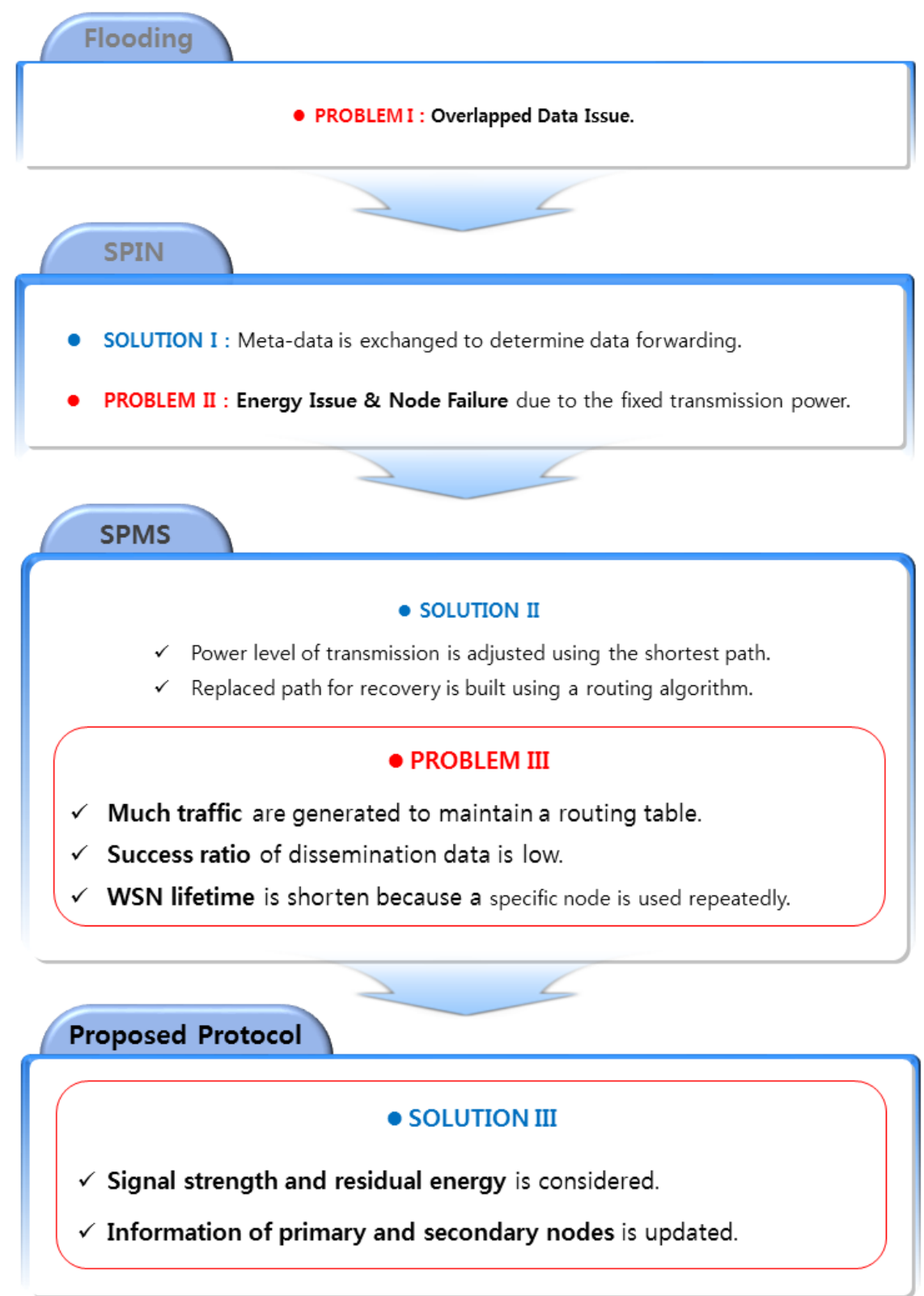

Fig. 1. Previous works and contributions of the proposed protocol

As stated above, effective power-consumption of data dissemination and connecting of sensor network are important issues in IoT-WSNs [25]. In this work, we are concerned with an energy-effective data-centric-based proactive dissemination protocol as flat type. In the proactive dissemination protocol, the sensed data is disseminated to the whole field, and our proposed protocol expressively prolongs initial disconnection time and makes certain of energy-efficient dissemination. Fig. 1 summarizes problems when data is disseminated to the 
entire wireless sensor network using prior schemes. There are much traffic of control messages, low success ratio of data transmission, and short network lifetime in SPMS. In order to overcome the mentioned weaknesses, we consider signal strength instead of the shortest path. Furthermore, we use current residual energy value to avoid node failure, and we keep the information of Primary and Secondary nodes with their residual energy values to recover dissemination path. Hence, our protocol could take into consideration the optimized power-consumption and disseminated time concurrently with no routing tables. Compared to our conference paper [26], in this work, we complete message types, and detailed operation with message flow chart for the proposed scheme in IoT-WSNs environment. In addition, more simulation results are analyzed.

In this paper, Section 2 explains how to work the conventional data distribution methods, SPIN and SPMS, and how to overcome the disadvantages of them. Section 3 presents details of our scheme with a case study of fault tolerance, and Section 4 demonstrates an excellent performance of the proposed scheme. Finally, Section 5 concludes this work.

\section{Related Work}

Flooding scheme and SPIN scheme [19] have been demonstrated for data dissemination. Flooding scheme does not need to employ a special structure to disseminate the sensed data. As shown in Fig. 2, the sensing data are repeatedly transmitted to all adjacent sensor nodes until it reaches the predetermined hop count. Sensor networking based on Flooding strategy is uncomplicated to be established, but it suffers from overlapped data problem [21].

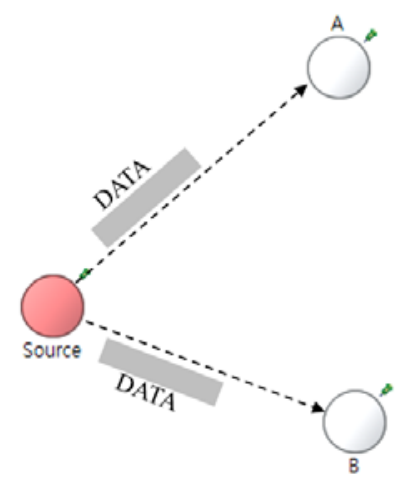

(a)

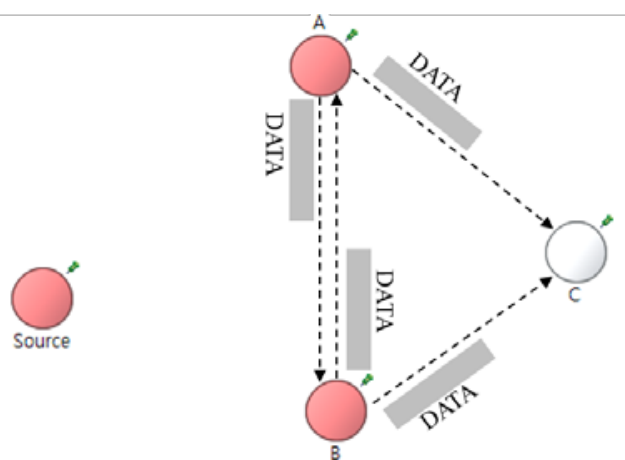

(b)

Fig. 2. Example of Flooding scheme: (a) Source sends data to neighbor node A and B;

(b) Node A and B transmit data to their neighbor nodes

Since a node sends data to all neighbor nodes regardless of whether the adjacent node already receives the necessary data, which leads to redundant power consumption, SPIN is designed to resolve the overlapped data issue [19]. Each node negotiates with its adjacent sensors before forwarding data, hence it is able to avoid transmitting unnecessary data. SPIN uses meta-data, which includes the contents of negotiation.

There are two kinds of dissemination protocols in SPIN. The first protocol is SPIN-PP and the second one is SPIN-BC; and, they use point-to-point (unicasting) and one-to-many (broadcasting) data transmissions, respectively. Hereafter, we only consider SPIN protocol as SPIN-PP. SPIN protocol has three phases, which are as follows. The first phase is the 
advertisement (ADV); a sensor having a data broadcasts an ADV advertisement message containing meta-data to its neighbors, as described in Fig. 3(a). The second phase is the request (REQ); when $\mathrm{ADV}$ message arrives at its neighbors, the neighbor analyzes the meta-data, then the need for data is shown, as depicted in Fig. 3(b). If the node requires the data, the neighbor sensor responds by sending an REQ request message back to the sender transmitted ADV message. The last phase is the data transmission; after the REQ message is transmitted, a sensor node forwards the requested data, as depicted in Fig. 3(c).

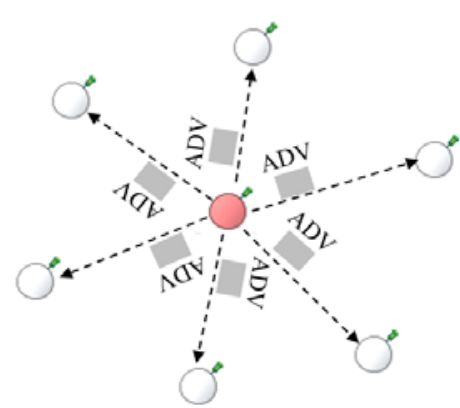

(a)

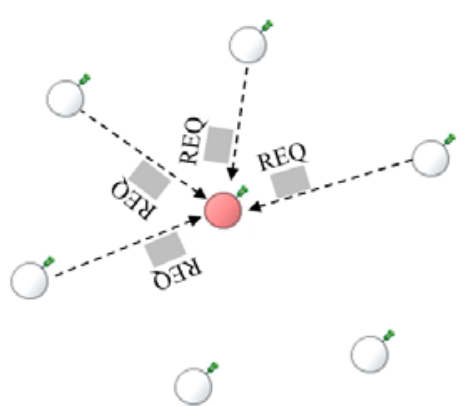

(b)

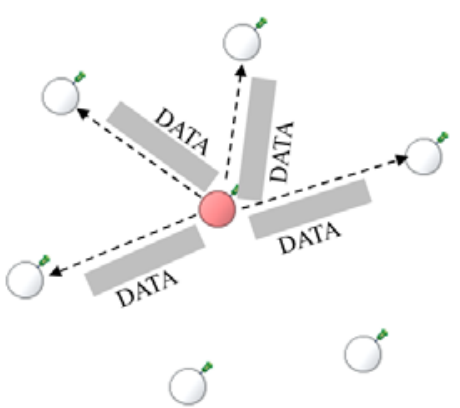

(c)

Fig. 3. Example of SPIN: (a) Advertisement step; (b) Request step; (c) Data transmission step

Although SPIN sends out a large number of redundant message compared to Flooding, these short meta-data messages come out relatively smaller than the sensed data [27]. The determination of the necessity to forward data is also simple, thus the computational overhead becomes negligible. However, SPIN shows the weakness of forwarding all packets using the fixed power without checking the range between adjacent nodes. Due to the power consumption model that data transmission increases exponentially with the distance described above, SPIN scheme might not be suitable for optimized data transmission. In order to overcome this drawback of SPIN, distance-based adjustment of transmission power has emerged as a major interest.

SPMS [23][24][28] protocol is designed from SPIN that exchanges information to determine data forwarding. SPMS employs a multi-hop manner to reduce the waste of power directly linked to aforementioned problem of SPIN. In the SPMS based operation, all destination nodes should be acknowledged before sending data, thus a routing table must be needed. Since hundreds of sensor nodes are composed in IoT-WSN, it is not efficient that each node creates its own routing information for whole network. Hence, every node just keeps the information for a constricted zone [29], which is set as a maximal transmission range of a sensor, to cut the cost for creating routing table. Here, each node establishes shortest paths for zone-members using Distributed Bellman-Ford (DBF) method beforehand. DBF algorithm, which is known as optimal, is executed based on distances between nodes [22]. When failure of certain sensor occurs on the using path, a replaced path for recovery is built.

In Fig. 4, a transmission cost is represented on each edge between sensors. The routing table of sensor C indicates that sensor B is primary node (called PRONE) and sensor A is secondary node (called SCONE) for destination sensor A. The primary node is chosen for energy-effective forwarding, and the secondary node is generally taken to recover a failure of the primary node. 


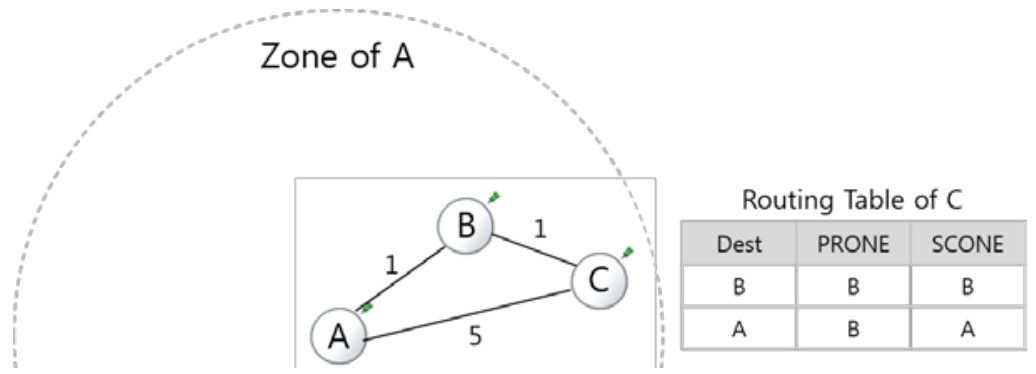

Fig. 4. Routing table of node C in SPMS

Authors of [30][31] evaluate their scheme (EDAS) in comparison with SPMS. EDAS expects an average distance between relay nodes using the actual distance between source and destination nodes with no routing information. However, the average distance could be not calculated because each node is not able to compute accurate distances, thus our results are only compared to the representative SPIN and SPMS schemes.

\section{Robust Energy Saving Data Dissemination Protocol}

As mentioned above, shortest path-based SPMS tries to minimize waste of transmission energy, however a specific node could be used repeatedly and WSN lifetime might be shorten. In addition, SPMS should continuously maintain routing table for each node, thus this might lead to much traffic and delay from DBF. In this paper, we design a dissemination protocol, which considers not only waste of transmission but also failure of sensor. There is no duplicated data, and any routing table is not needed. For these reasons, our scheme could be suitable in IoT-WSN environment.

\subsection{Components for the Proposed Protocol}

ADV, REQ, ACK messages and $\mathrm{T}_{\text {Adv }}$ and $\mathrm{T}_{\text {Data }}$ timers are described as follows. Every sensor keeps only two information; Primary and Secondary sensors.

- Advertisement (ADV) message: A node, having a data, broadcasts ADV to check whether the data is necessary for neighbor nodes. First, the source node, which initially senses the data, inserts current its residual energy value and the origin of the data into $A D V$. Second, every relay node, having a received data, inserts its current residual energy and the data sender's residual energy information retrieved from ACK into ADV.

- Request (REQ) message: If a node requires the data, the node sends REQ to the neighbor which has broadcasted ADV.

- Acknowledge (ACK) message: When REQ is transmitted to a node from neighbors, the node sends ACK back to the REQ senders. The ACK also has the information of its current residual energy. After that, the data could be transmitted to the REQ senders.

- Timer $\mathbf{T}_{\text {Adv }}$ : After ADV is received, the node sets a timer $\mathrm{T}_{\text {Adv }}$ instead of sending an REQ. $\mathrm{T}_{\mathrm{Adv}}$ should be assigned according to the signal strength from the ADV sender. For example, if the strength value is high, the timer value must be shorter than that of weak 
strength. If timer $\mathrm{T}_{\mathrm{Adv}}$ expires, then REQ is sent to Primary node. On the other hand, REQ is overheard from neighbors, then own timer $\mathrm{T}_{\mathrm{Adv}}$ has to be terminated.

- Timer $\mathbf{T}_{\text {Data: }}$ : A value of timer to assure data receiving from Primary node, $T_{\text {Data }}$, should be assigned as a maximal wait time. $\mathrm{T}_{\text {Data }}$ is fixed after REQ is sent. If the requiring data is not obtained till timer is finished, another REQ is resent to Secondary node once more.

- Primary and Secondary Nodes: A node has the information of residual powers of Primary and Secondary nodes. Whenever new ADV or ACK messages are received, new residual energy values in messages are compared to current values. Primary node is the neighbor node, which has the maximal residual energy value of neighbors, and Secondary node is another neighbor node, which has the maximal remained energy value of neighbors except Primary node.

\subsection{Message Flow Chart}

Fig. 5 explains the message flows in case of four nodes in a zone area. To disseminate the sensed data, the source node broadcasts an ADV message including residual energy value to three neighbor nodes. The neighbor nodes, which have received the ADV, register its information of Primary and Secondary nodes using the residual energy value in the ADV. Each neighbor node sets up its timer $\mathrm{T}_{\text {Adv }}$ based on the signal strength from the source node.

When timer $\mathrm{T}_{\text {Adv }}^{1}$ of neighbor node 1 expires, node 1 transmits REQ to source node to receive the data. Simultaneously, node 1 creates its timer $T^{1}$ Data. Since neighbor nodes 2 and 3 are aware of REQ transmission, the timers $\mathrm{T}^{2}{ }_{\text {Adv }}$ and $\mathrm{T}^{3}{ }_{\mathrm{Adv}}$ are terminated. When REQ is received from node 1 at source node, it sends an ACK including its residual energy value back to node 1 . Sequentially, the sensing data of source node should be disseminated to node 1 .

If the node 1 receives ACK, it updates the Primary and Secondary information using the residual energy information in ACK message. The node 1 broadcasts an ADV including its residual energy. Neighbor nodes 2 and 3 update their Primary and Secondary information using ADV received from the node 1 . They set their timer $\mathrm{T}_{\text {Adv }}$ based on the measured signal strength from the node 1.

If timer $\mathrm{T}^{2}$ Adv of neighbor node 2 is completed, REQ is sent to node 1 , and timer $\mathrm{T}_{\text {Data }}^{2}$ is set at once. Since neighbor node 3 overhears REQ, timer $\mathrm{T}^{3}$ Adv is also terminated. When node 1 receives REQ from node 2, node 1 sequentially sends an ACK including its residual energy and data back to node 2. After ACK is received from node 1 at node 2 , timer $\mathrm{T}^{2}{ }_{\text {Data }}$ is terminated and Primary and Secondary information are also updated.

At the same way, node 2 broadcasts an ADV. Node 3 sets up timer $\mathrm{T}^{3}$ Adv and updates Primary and Secondary information using ADV received from node 2. As timer $\mathrm{T}^{3}$ Adv is finished, node 3 forwards REQ. Node 2 sends ACK and the received data to node 3 . Node 3 updates Primary and Secondary information, and the data is finally disseminated to all nodes. 


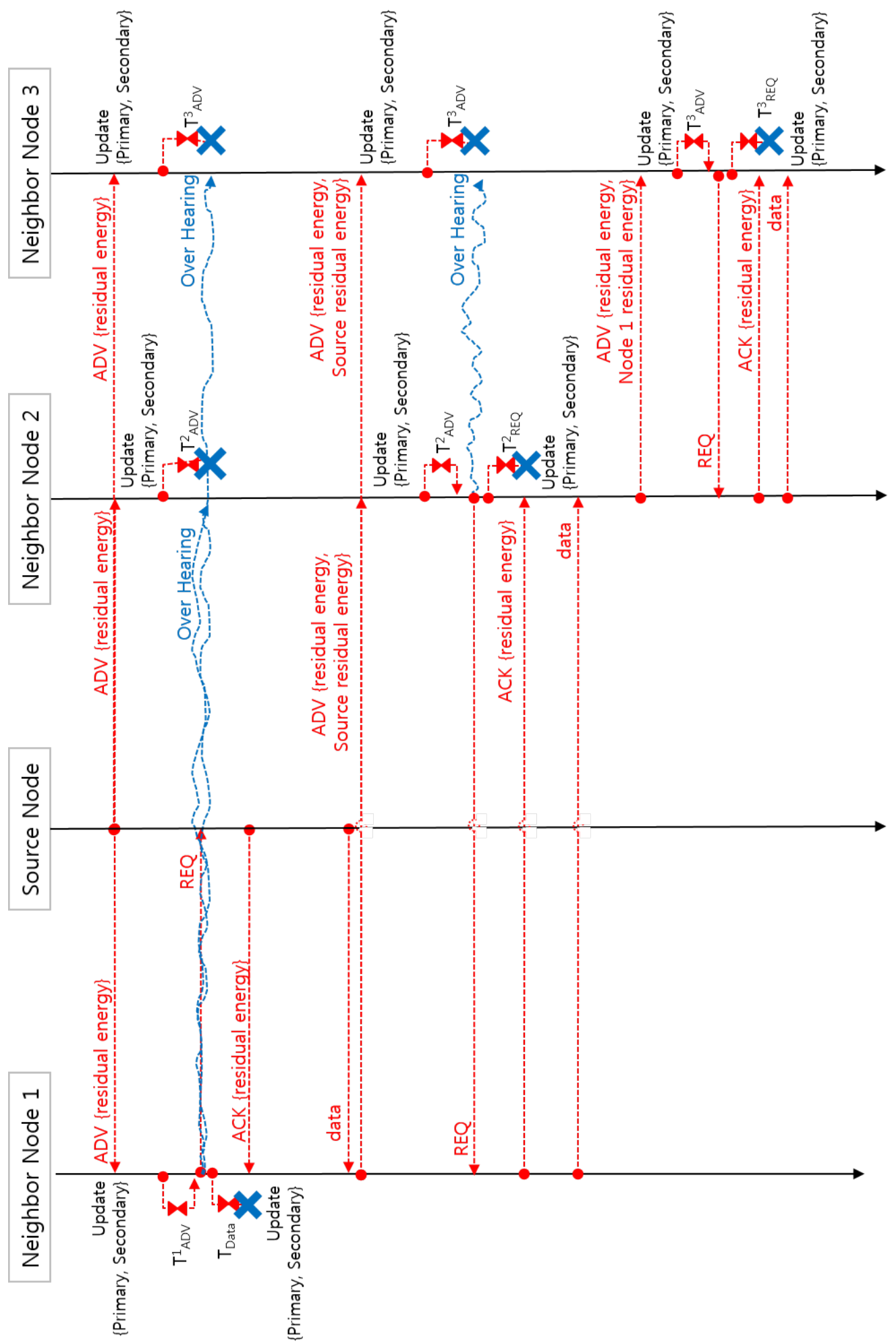

Fig. 5. Example of the proposed scheme: message flow chart 


\subsection{A Case Study of Fault Tolerance}

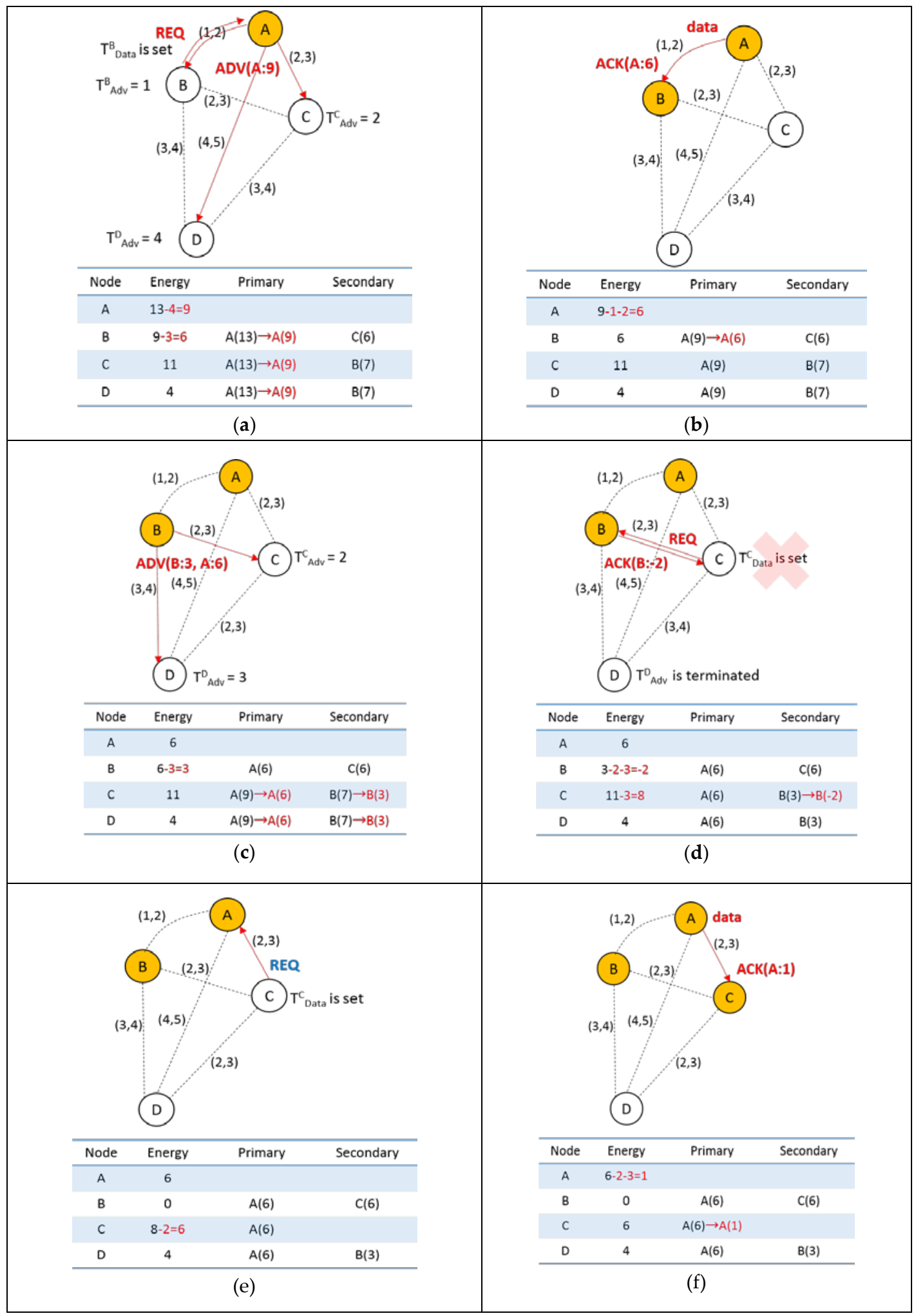

Fig. 6. Example of recovery from failure of node B 
Fig. 6 explains an example of fault tolerance in our proposed protocol. There are four nodes in the zone of node A, and each wireless link has costs associated with energy consumptions as a pair (control message transmission, data transmission). We assume that each node is able to measure its current residual energy, and the value of each link could depend on the distance between nodes [22]. In Fig. 6(a), the initial residual energy values of nodes A, B, C, and D are assumed to be $13,9,11$, and 4 , respectively to explain a case of fault tolerance. The information of Primary and Secondary for node A is void, initially. For an example of the information of node B, the information of Primary is A(13), and it means that the Primary of node B is node A and node A's residual energy is 13 until now. In Fig. 6(a), to send the sensed data in source node A, node A first broadcasts ADV message including its current residual energy value of $\mathrm{A}$ in its zone. Since the maximum energy consumption (i.e., maximum transmission radius) of ADV control message transmission is 4, here we may think the transmission from $\mathrm{A}$ to $\mathrm{D}$, the included value in ADV is $9(=13-4)$. After all nodes in zone of A receive the $A D V$ message, nodes $B, C$, and $D$ update their current energy information of Primary node A as 9, which is denoted by A(9). Source node A also updates its residual energy value as 9 . They set up their timer $\mathrm{T}_{\mathrm{Adv}}$ based on the signal strengths from node $\mathrm{A}$. Timer $\mathrm{T}^{\mathrm{B}}{ }_{\mathrm{ADV}}$ of node $\mathrm{B}$ first expires, and node $\mathrm{B}$ transmits REQ message to node $\mathrm{A}$ using maximum energy consumption of control message, 3. Simultaneously, node $\mathrm{B}$ sets up its timer $\mathrm{T}^{\mathrm{B}}$ Data, and current residual energy of node $B$ is changed as $6(=9-3)$. Since neighbors $C$ and $D$ are aware of the REQ transmission, their timers $\mathrm{T}_{\mathrm{Adv}}$ are terminated. As node A gets the REQ message from node B in Fig. 6(b), node A sends ACK message including its residual energy 6 (=9-1-2) back to node B. Sequentially, node A transmits the sensed data, and its residual energy value is updated. After node B receives the ACK, the energy information of Primary node A is updated as 6, denoted by $\mathrm{A}(6)$. In Fig. 6(c), node $\mathrm{B}$ broadcasts $\mathrm{ADV}$ message including its residual energy value $3(=6-3)$ and the received energy value of node $A$, denoted by $\operatorname{ADV}(B: 3, A: 6)$, to send the received data. Here we may think the maximum transmission energy from $\mathrm{B}$ to D. When nodes $C$ and $D$ receive the ACK message, they set up their timers $T_{\text {Adv }}$ based on the signal strengths from node $B$. They also update their energy information of Primary and Secondary nodes as A(6) and B(3), respectively.

In Fig. 6(d), since the timer $T^{C}{ }_{\text {Adv }}$ of Fig. 6(c) is earlier expired than $T^{D}$ Adv, node $C$ sends REQ message to node $B$ using maximum energy consumption of control message, 3 . At the same time, node $\mathrm{C}$ sets up timer $\mathrm{T}^{\mathrm{C}}$ Data and updates its residual energy value as $8(=11-3)$. Since node $\mathrm{D}$ overhears the REQ message, its timer $\mathrm{T}^{\mathrm{D}}$ Adv is terminated. As node $\mathrm{B}$ receives the REQ, and it sends ACK message including its residual energy $-2(=3-2-3)$ back to node $C$. In detail, node $\mathrm{C}$ is thinking that it is able to receive the data from node $\mathrm{B}$ up to now, however node $\mathrm{B}$ would be failure after sending ACK message and the data. To figure out this one in our protocol, the expected residual energy -2 should be included in ACK message. Since node C checks the value -2 , it could expect a failure of node $B$; thus node $C$ terminates its timer $T^{C}{ }_{\text {Data }}$. In Fig. 6(e), node $C$ tries to send REQ (colored by blue) message to node A using Primary node information, and it updates residual energy value $6(=8-2)$. Here, node $C$ need not to use maximum energy consumption because it uses information of Primary. Node $\mathrm{C}$ directly sends the REQ to its Primary node A using energy consumption of control message, 2. At the same time, it also sets up timer $\mathrm{T}^{\mathrm{C}}$ Data. In Fig. 6(f), when node A gets the REQ message from node $\mathrm{C}$, it sends ACK message including its residual energy $1(=6-2-3)$ back to node $C$, sequentially the data is transmitted. After node $\mathrm{C}$ receives the ACK, the energy information of Primary node A is updated as 1 . In this way as above, node $\mathrm{C}$ dispatches the received data to node $\mathrm{D}$ without failure of node $\mathrm{B}$. 


\subsection{An Example of Multiple sources}

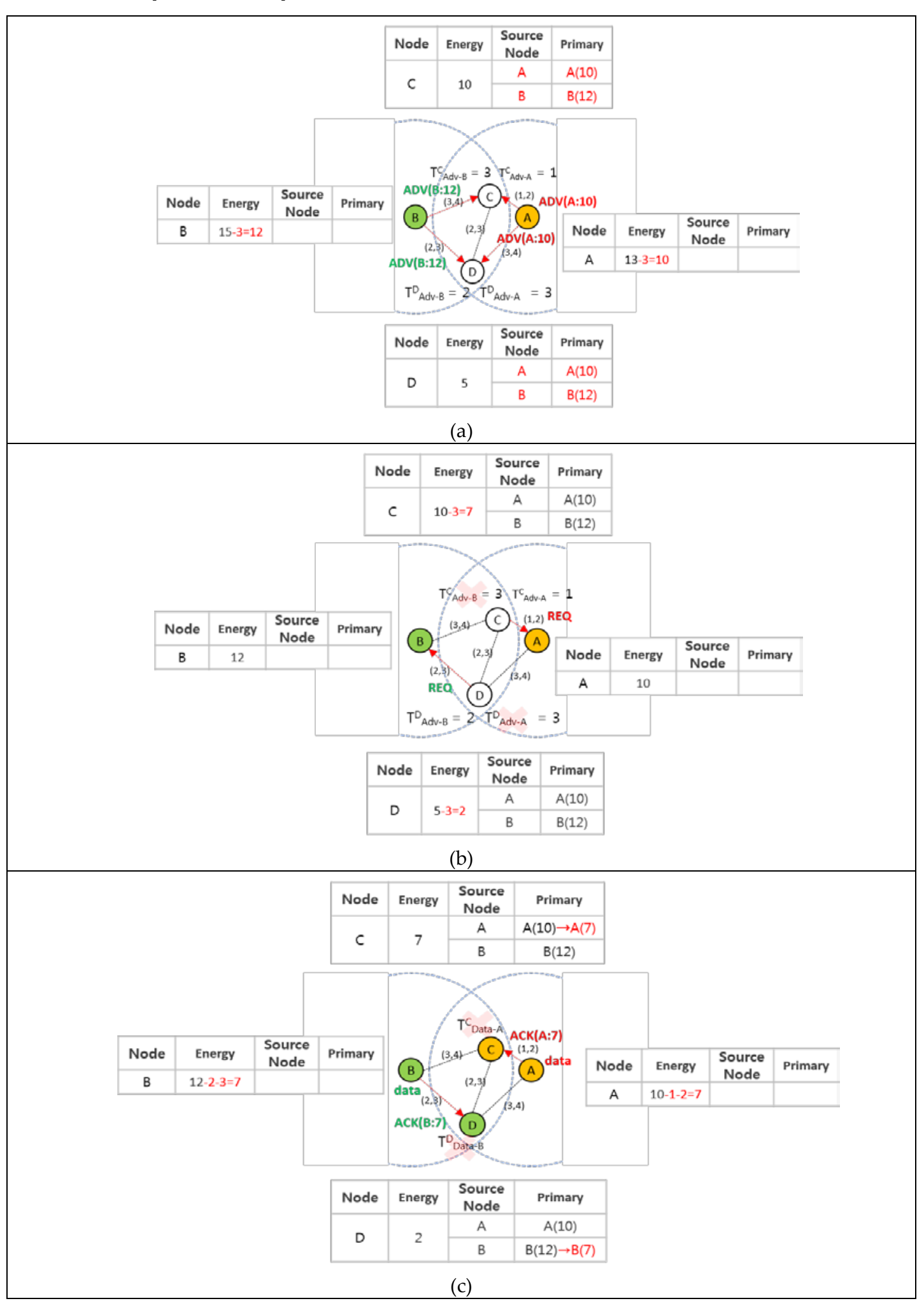

Fig. 7. Example of disseminating data sensed from multiple sources 
Fig. 7 explains an example of disseminating data sensed from multiple sources. In brief, each node has multiple information of Primary and Secondary for multiple sources, separately. For each source node, each data could be disseminated to the whole network as mentioned above.

As shown in Fig. 7(a), we assume that a source node A is not in zone of B, and another source node B is not in zone of A, either. In addition, Nodes C and D are in intersection of zones of A and B. In a similar way above, each wireless link has costs associated with energy consumptions. Current residual energy values of nodes A, B, C, and D are assumed to be 13, 15, 10, and 5, respectively. Nodes A and B have sensed data in different places, and each source node first broadcasts ADV message in each zone. Since the maximum energy consumption of ADV control message for source A is 3, the included residual energy value in $\mathrm{ADV}$ is 10 (=13-3). Moreover, the origin of the data is indicated as source A. In the same way, node $\mathrm{B}$ broadcasts ADV control message, which includes the value $12(=15-3)$ and the origin of data as source B. Node C and D receive ADV messages from node A and B, and they generate the information of Primary from source $A$ and $B$, which are denoted by $A(10)$ and $\mathrm{B}(12)$, using the included values of $\mathrm{ADV}$ messages. At the same time, node $\mathrm{C}$ and $\mathrm{D}$ set up their timers, based on the signal strengths from all sources, for ADV messages. For instance, node $\mathrm{C}$ sets up the timer, $\mathrm{T}^{\mathrm{C}}{ }_{\text {Adv-A }}$, for $\mathrm{ADV}$ from source $\mathrm{A}$.

In Fig. $7(\mathbf{b}), \mathrm{T}^{\mathrm{C}}$ Adv-A of node $\mathrm{C}$ first expires in zone of $\mathrm{A}$, and node $\mathrm{C}$ transmits REQ message using maximum energy consumption of control message, 3 . Thus, current residual energy of node $C$ is changed as $7(=10-3)$. At this time, neighbor $D$ is aware of REQ transmission, and its timer $\mathrm{T}_{\text {Adv-A }}^{\mathrm{D}}$ is terminated. In the same manner, current residual energy of node D is updated as 2 (=5-3), and node C's timer $\mathrm{T}_{\text {Adv-B }}^{\mathrm{C}}$ is terminated. In Fig. 7(c), nodes $\mathrm{C}$ and $\mathrm{D}$ set up $\mathrm{T}^{\mathrm{C}}{ }_{\text {Data-A }}$ and $\mathrm{T}^{\mathrm{D}}{ }_{\text {Data-B }}$ after broadcasting REQ messages, respectively. As source A gets the REQ message from node $\mathrm{C}$, it sends ACK message including its residual energy 7 (=10-1-2), and the sensed data back to node $C$, then node A's residual energy value is updated. In the same way, ACK message and data of source B are consecutively transmitted to node $\mathrm{D}$, and residual energy value of node $B$ is changed 7 (=12-2-3). After node $C$ receives the ACK, the energy information of Primary node $A$ is updated as $A(7)$. Node $D$ also updates its energy information of Primary node B as B(7). Even though we do not describe data dissemination processes any more, it seems to be enough to explain dissemination operation for multiple sources. The rest of operation is same manner as mentioned flow chart and case study.

\section{Simulation Results and Analysis}

The proposed dissemination scheme is implemented using well-known network simulator Qualnet [32][33] to analyze its efficiency compared with mentioned schemes under single and multi-zone environments.

\subsection{Analysis for Single-zone Environment}

The main parameters for single-zone environment are listed in Table 1, and Fig. 8 shows an example for 25 IoT-sensor nodes in a grid type of network over area $150 \mathrm{~m} \times 150 \mathrm{~m}$. A source node is in the bottom-left, and it generates 1,000 sensed data continuously under CBR. Every node has abundant initial energy, and all data would be disseminated to the whole network using mentioned protocols. We can measure average energy consumption and average transmission time after termination of the simulation. The average energy consumption means that each node may consume energy on average until all data are disseminated to the entire 
network, and it is calculated as sum of all nodes' energy consumptions is divided by number of nodes. In the same way, the average transmission time is an expected dissemination time for each node, and it is calculated as sum of each node's spent time is divided by number of nodes.

Table 1. Simulation environment in single-zone

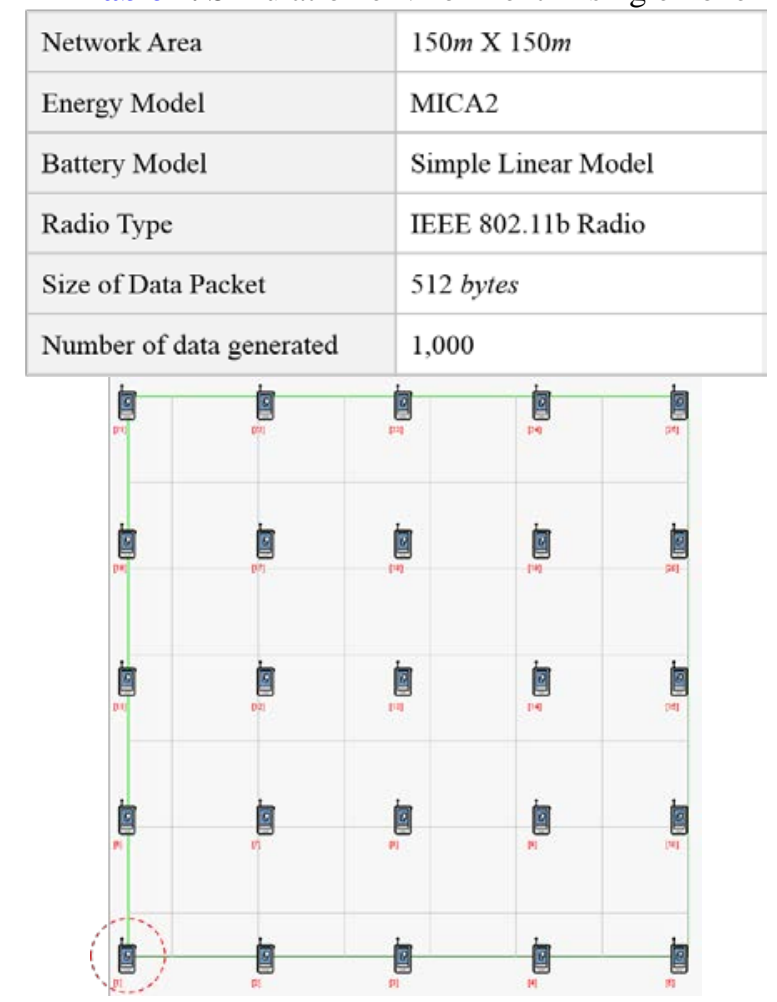

Fig. 8. An example for a given network, 25 IoT-sensor nodes for single-zone

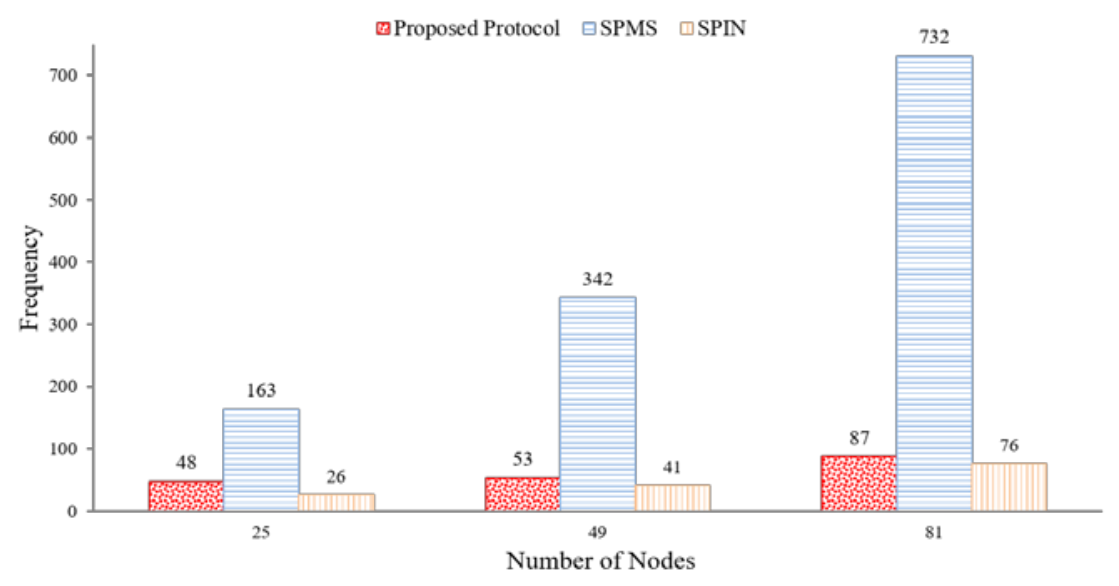

Fig. 9. Average count of control packets

First, we analyze average count of control packets in terms of several sensor nodes. We assume that numbers of sensors are 25,49 , and 81 . Here, the number of sensors means 
node-density for the given network size. As shown in Fig. 9, SPMS generates many control messages due to DBF. Since our protocol does not use DBF, the efficiency for the count of control packets increases to approximately $71 \%, 85 \%$, and $88 \%$ as compared with SPMS. Here, we assume that the size of control packets is 4 Bytes in this simulation, however there is no need to be restricted as the size. Fig. 10 shows that SPMS consumes more energy for transmitting control messages than those of others. Here, average energy consumption is calculated as sum of all nodes' energy consumptions is divided by the number of nodes. Although the values of SPMS are small, many control packets could lead to traffic congestion in IoT-WSNs. SPIN uses a meta-data to avoid data duplication, and we also similarly ADV and REQ messages. Thus, the proposed protocol and SPIN are pretty much the same in number of control messages. However, our protocol could be flexible in responding to failure of sensor node due to the control message including residual energy information.

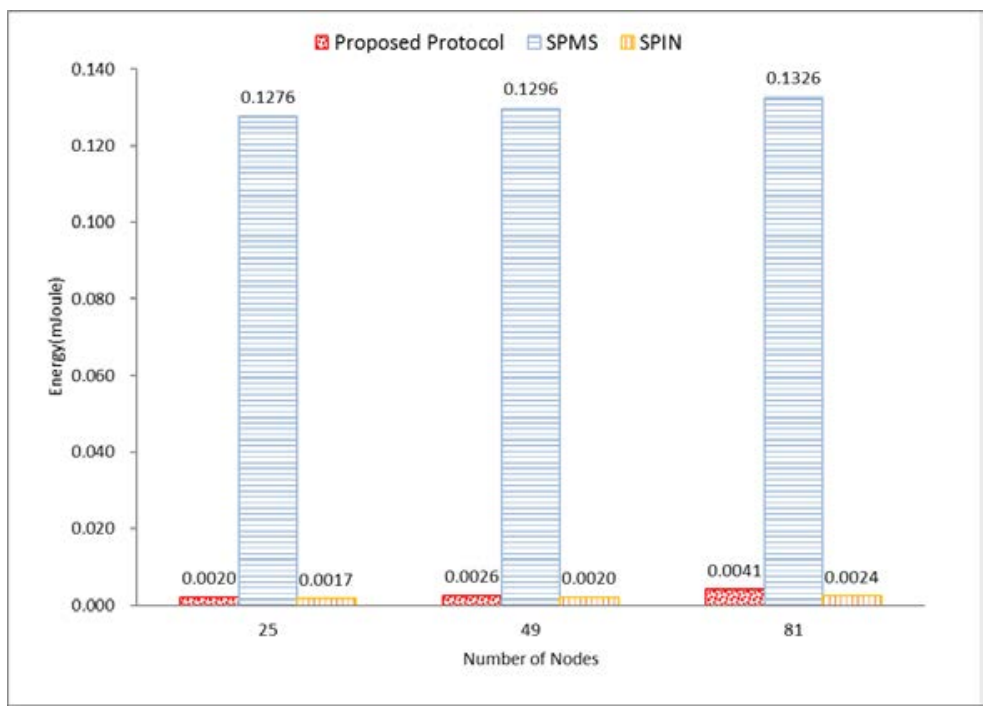

Fig. 10. Average energy consumption for control packets

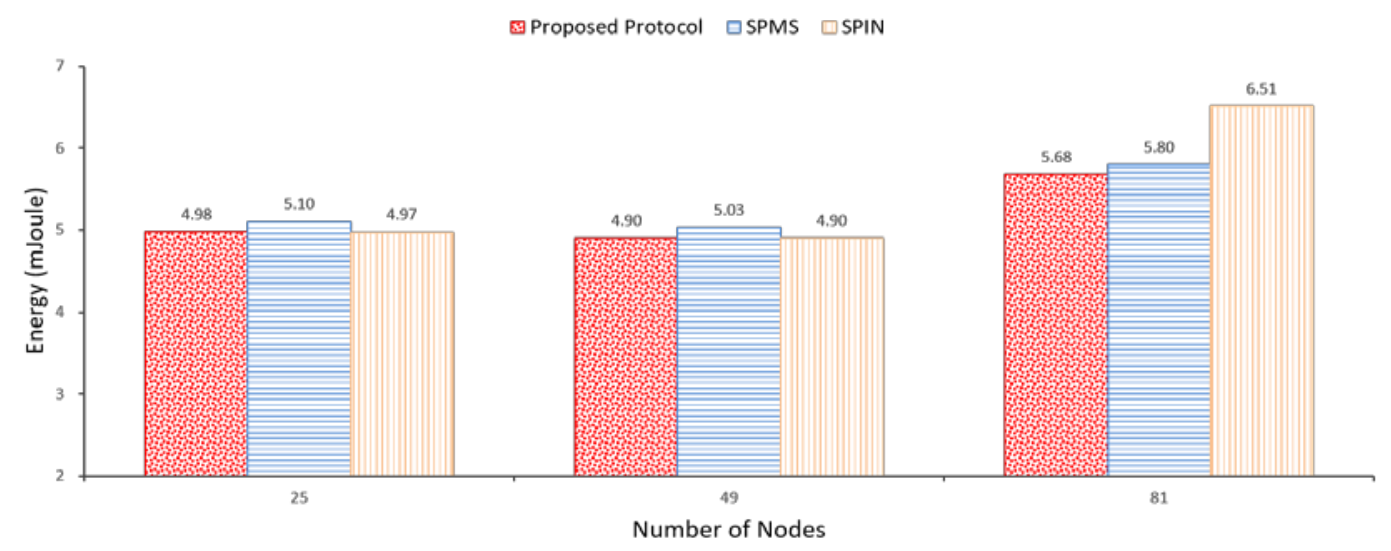

Fig. 11. Average energy consumption for data transmission

Energy consumptions of the proposed protocol are similar to those of SPMS, since they use distance to neighbor nodes through adjusting transmission power. Although single-zone 
environment is considered, if the node-density is high (81 sensors) as indicated in Fig. 11, then the efficiency for power-consumption increases to about $13 \%$ in comparison with SPIN.

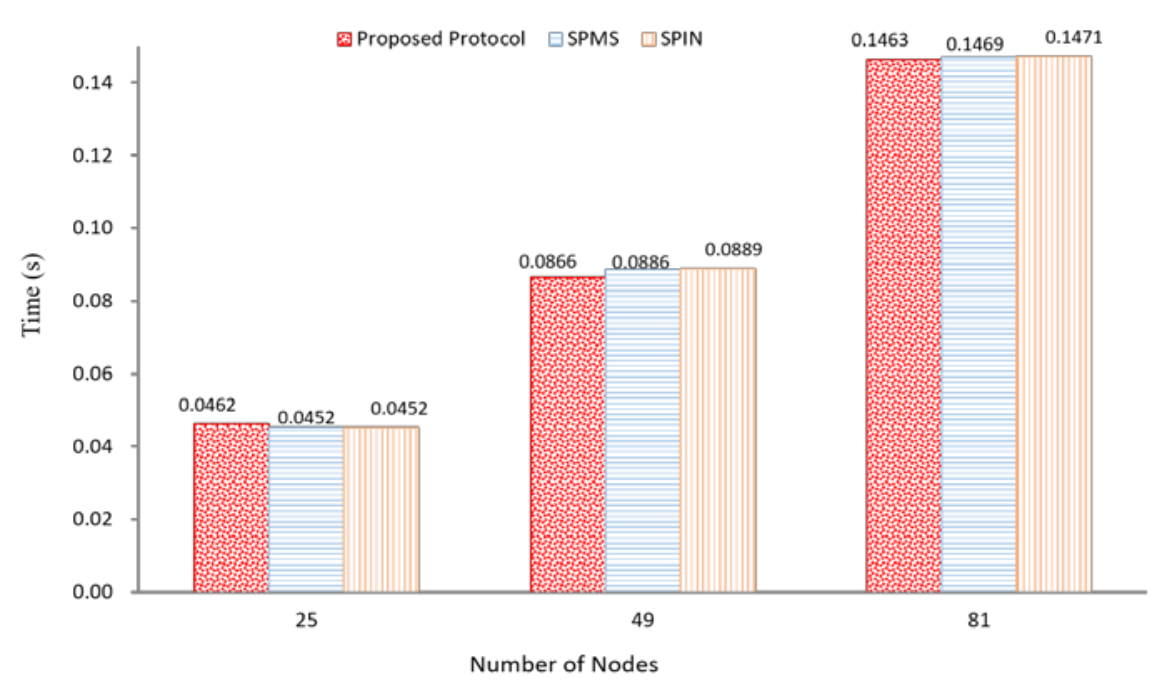

Fig. 12. Average transmission time until all data are disseminated to whole network

Finally, Fig. 12 explains the average transmission time total data to whole network. Here, transmission times of all schemes are similar due to single-zone. Later we will show and discuss that the proposed protocol and shortest path-based SPMS outperform SPIN in Fig. 14 for multi-zone.

\subsection{Analysis for Multi-zone Environment}

The main parameters for multi-zone environment are listed in Table 2. We assume that each sensor node has enough initial energy and a source node also generates 1,000 data continuously as the single-zone. The data would be disseminated to the whole network using three protocols.

Table 2. Simulation environment in multi-zone

\begin{tabular}{|l|l|}
\hline Network Area & $550 \mathrm{~m}$ X $550 \mathrm{~m}$ \\
\hline Energy Model & MICA2 \\
\hline Battery Model & Simple Linear Model \\
\hline Radio Type & IEEE $802.11 \mathrm{~b}$ Radio \\
\hline Size of Data Packet & 512 bytes \\
\hline Number of data generated & $1,000 /$ Infinity \\
\hline
\end{tabular}

In Fig. 13, we note that energy consumptions of our protocol are similar to those of SPMS because they consider transmission power to send data. SPMS obtains the shortest paths to disseminate data using DBF, thus it seems to consume transmission energy optimized. That is, the proposed protocol of Fig. 13 also approaches to optimal transmission energy consumption. Since SPIN uses same transmission power as mentioned above, our protocol outperforms up to 
$31 \%, 30 \%$, and $20 \%$ in comparison with SPIN. Moreover, our scheme works like shortest path-based SPMS, and Fig. 14 explains that the efficiency increases up to 3.7\% and 10.1\% in comparison with SPIN as distinct from Fig. 12 for single-zone. Actually, because transmission time has to depend on distance, if the shortest path or something like that is used, then dissemination delay could be optimized. Up to now, we only consider environments, which have no failure of sensor nodes. In the next subsection, we constrict the initial energy value of each sensor node to generate some node failures.

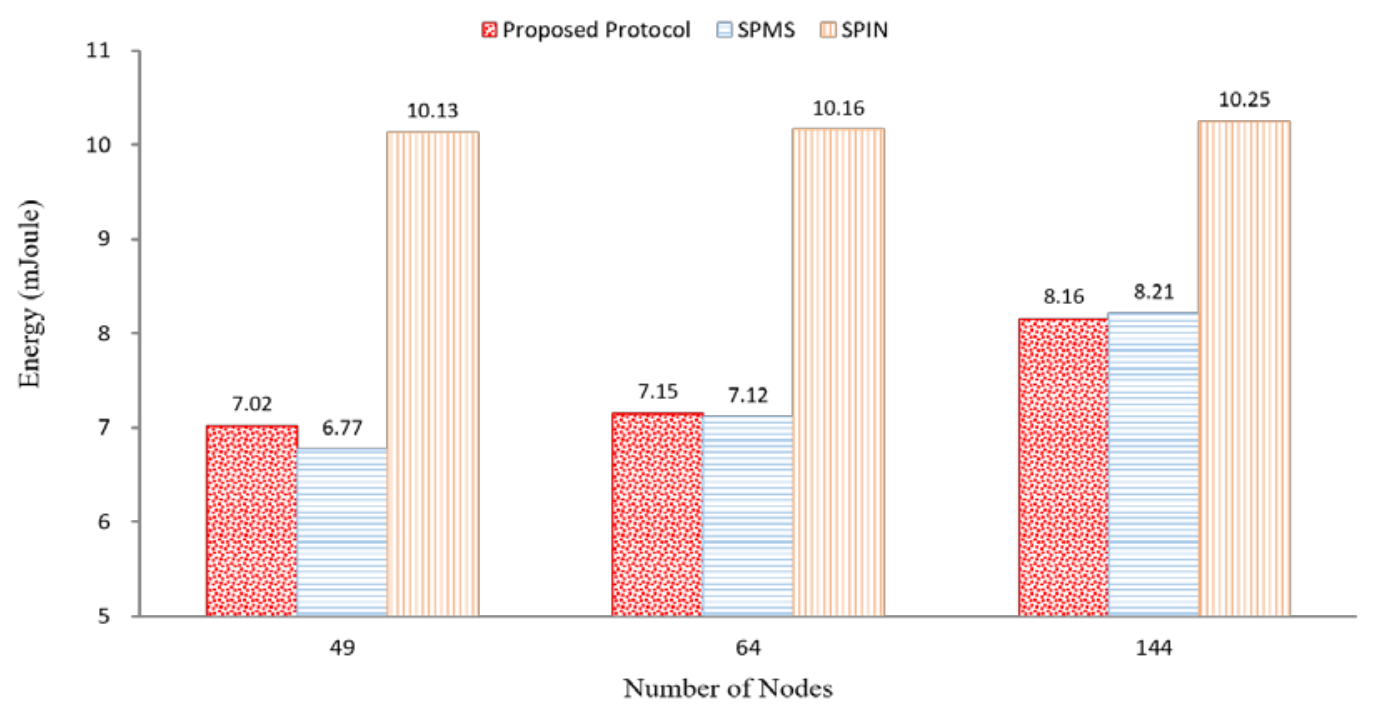

Fig. 13. Average energy consumption for data transmission

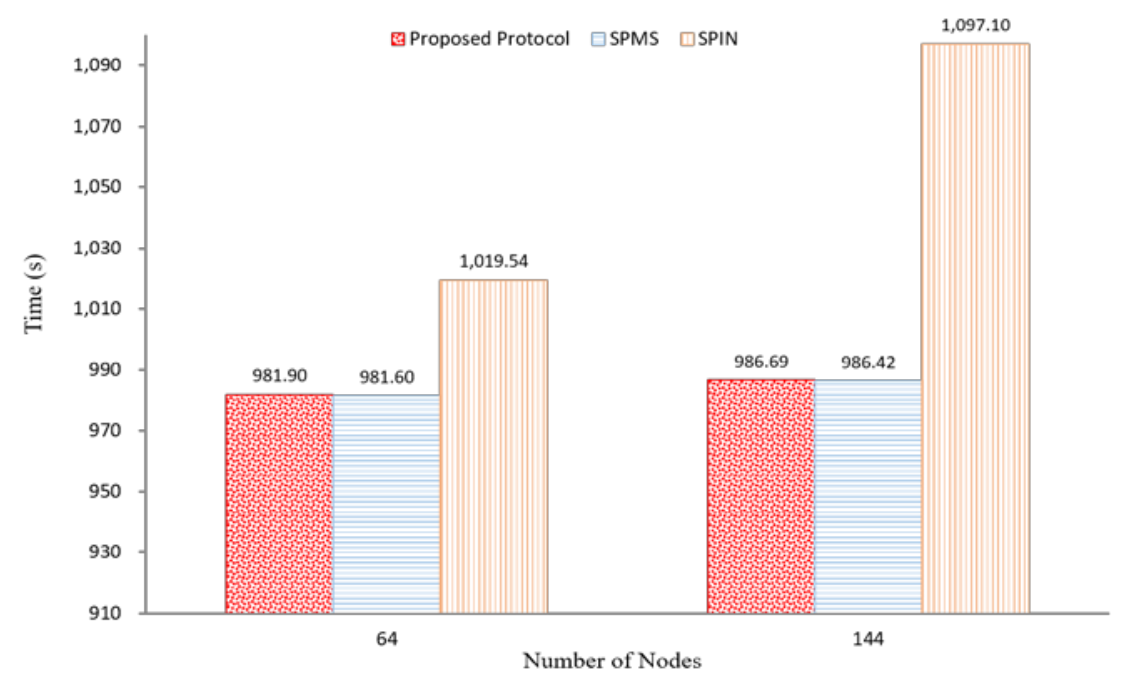

Fig. 14. Average transmission time until all data are disseminated to whole network under multi-zone environment 


\subsection{Analysis for Fault Tolerances}

The success ratio means the number of nodes, which are successfully received the transmitted data from the source node, over all nodes number until the network is disconnected in a multi-zone environment. In addition, the success ratio is not considered in a single-zone environment because all nodes are guaranteed to receive the data from the source node. In Table 2, the initial value of stored energy at every node is assigned as 5 mJoule except for source node in a multi-zone environment. To measure average success ratio for disseminated data, the source node generates data packets infinitely by the earliest network disconnection. If the energy of a node is exhausted, the node is considered to be a fault node. Actually a node failure of SPMS would be caused by using the shortest path repeatedly; thus, to some degree, we could expect the success ratio in comparison with our protocol.

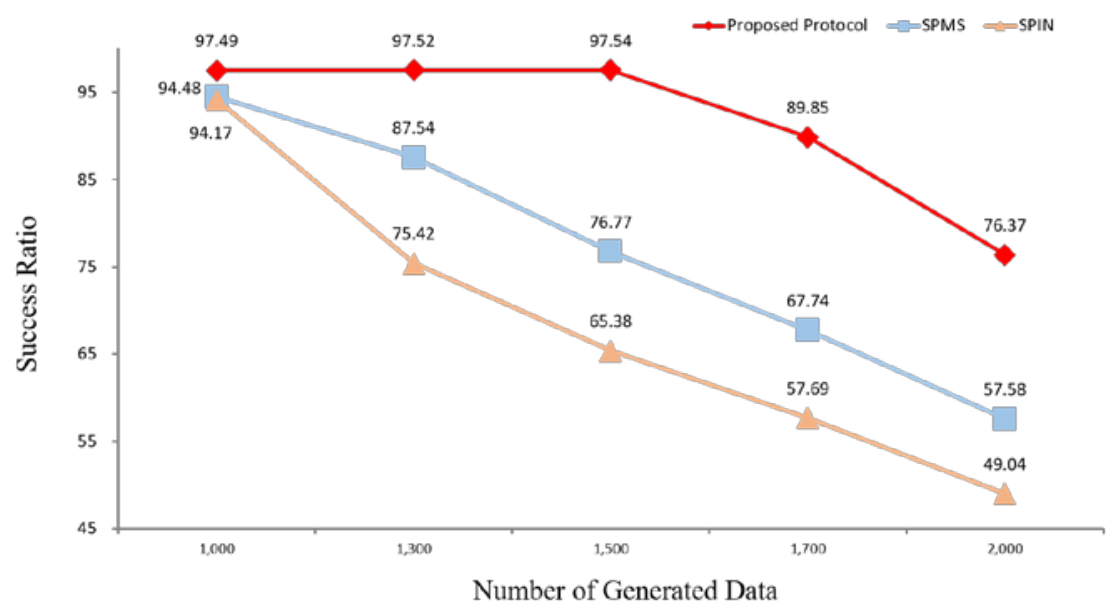

(a)

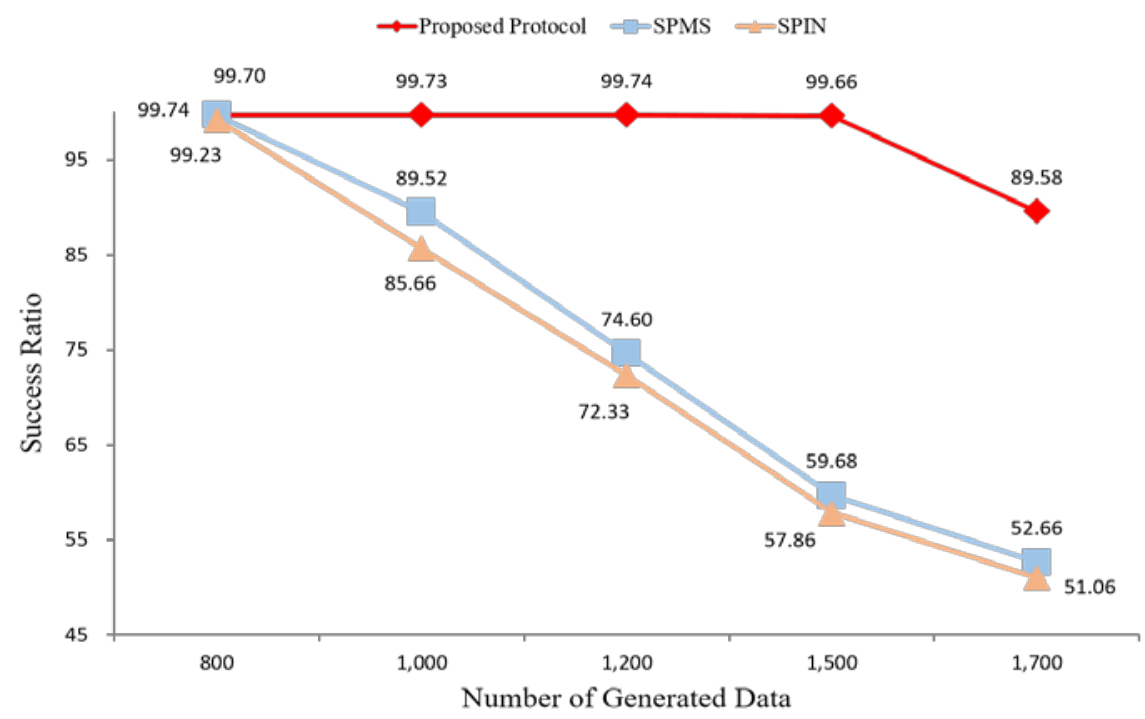

(b)

Fig. 15. Average Success Ratio of Transmission Data: (a) 49 Nodes; (b) 64 Nodes 


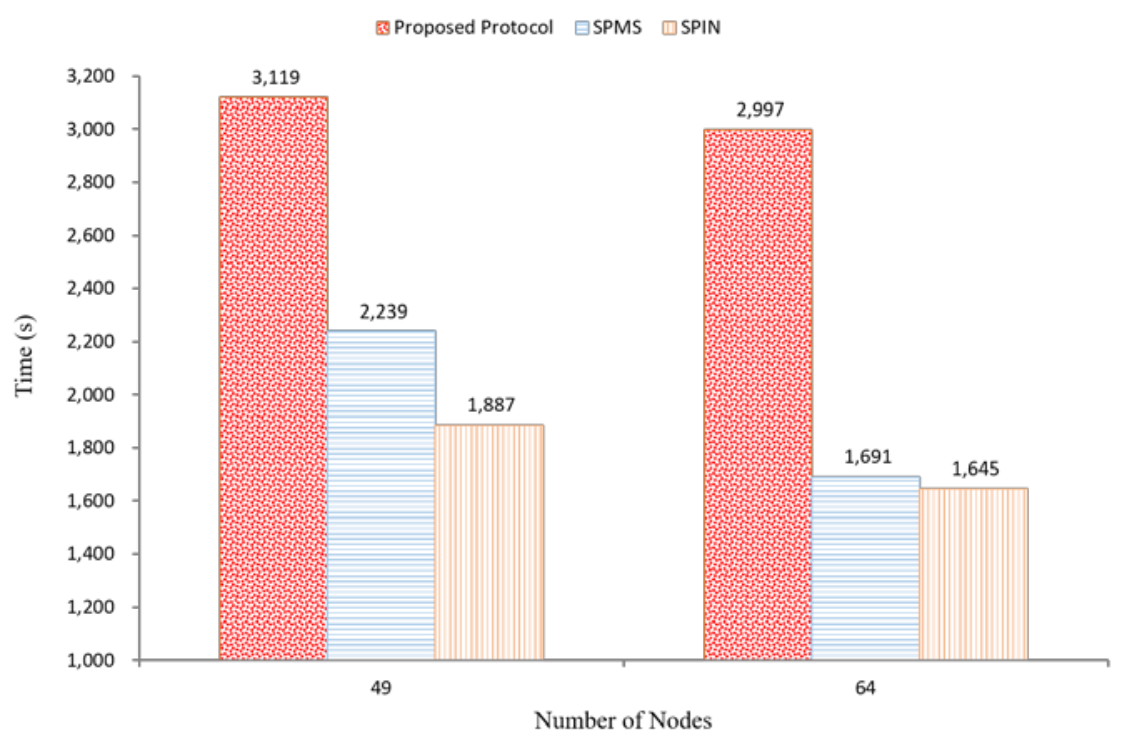

Fig. 16. Initial Disconnection Time

Fig. 15 explains average success ratios for each protocol and several nodes - (a) 49 and (b) 64. Our scheme considers both remaining energy and power consumption of sensor. And routing table is not necessary, thus it outperforms previously proposed schemes. Furthermore, since the network disconnection time of our scheme is much more extended than those of other protocols as shown in Fig. 16, the lifetime of our protocol outperforms those of SPMS and SPIN. As mentioned above, the proposed protocol and SPMS have similar results in terms of average energy consumption for data transmission as shown in Fig. 13. Actually, there is no node failure in the environments of Fig. 13. However, SPMS keeps using specific nodes on the shortest paths repeatedly, thus it could lead to node failures. On the other hand, our protocol considers residual energy of every node, thus a deviation in energy consumption for every node has to be lower than that of SPMS. In our simulation, we assume that the size of data packet is 512 Bytes. If the size is bigger than it, then some node failures of SPMS could occur earlier and/or more frequently than the current case. Therefore, we are able to expect that success ratio and initial disconnection time are lower and faster than Fig. 15 and Fig. 16, respectively. Hence, we note that our protocol could be appropriate for IoT-WSNs, which has a most fundamental energy problem.

\section{Conclusion}

In the early part of this paper, we have investigated various data dissemination protocols for IoT-WSNs environment. Since the energy-efficiency and network lifetime problems are important issues, our proposed dissemination protocol has taken remained energy and signal strength into account for disseminating data. The proposed protocol has outperformed well-known SPIN and SPMS in terms of total count of control packets, total energy consumption, transmission time, data transmission success ratio, and the earliest wireless sensor network disconnection time for single-zone and multi-zone environments. Even though SPMS establishes shortest path-based routing information by DBF, dissemination time of SPMS is not much different from our protocol. Our protocol also does not need to have a routing table, hence the count of control packets of our scheme is much less than that of SPMS. 
Finally, since the life time of our protocol is longer than those of SPIN and SPMS, we note that the proposed protocol could be more appropriate than other protocols for IoT-WSNs.

\section{Acknowledgement}

This work was supported by Incheon National University Research Grant in 2017.

\section{References}

[1] S. Li, L. D. Xu, and S. Zhao, “The internet of things: a survey,” Springer, Information Systems Frontiers, vol. 17, iss. 2, pp. 243-259, April 2015. Article (CrossRef Link)

[2] S. K. Lee, M. Bae, and H. Kim, "Future of IoT Networks: Survey," MDPI, Applied Sciences, vol. 7, no. 10, 1072, 2017. Article (CrossRef Link)

[3] P. Gonizzi, G. Ferrari, V. Gay, and J. Leguay, "Data dissemination scheme for distributed storage for IoT observation systems at large scale," Elsevier, Information Fusion, vol. 22, pp. 16-25, March 2015. Article (CrossRef Link)

[4] C.-Y. Chang, C.-H. Kuo, J.-C. Chen, and T.-C. Wang, "Design and Implementation of an IoT Access Point for Smart Home,” MDPI, Applied Sciences, vol. 5, no. 4, pp. 1882-1903, 2015. Article (CrossRef Link)

[5] I. Yaqoob, E. Ahmed, I. A. T. Hashem, A. I. A. Ahmed, A. Gani, M. Imran, and M. Guizani, "Internet of Things Architecture: Recent Advances, Taxonomy, Requirements, and Open Challenges,” IEEE Wireless Communications, vol. 24, iss. 3, pp. 10-16, June 2017.

Article (CrossRef Link)

[6] P. P. Ray, M. Mukherjee, and L. Shu, "Internet of Things for Disaster Management: State-of-the-Art and Prospects,” IEEE Access, vol. 5, pp. 18818-18835, September 2017. Article (CrossRef Link)

[7] H. Yetgin, K. T. K. Cheung, M. El-Hajjar, and L. Hanzo, "A Survey of Network Lifetime Maximization Techniques in Wireless Sensor Networks," IEEE Communications Surveys \& Tutorials, vol. 19, no. 2, pp. 828-854, January 2017. Article (CrossRef Link)

[8] M. Z. Hasan and F. Al-Turjman, "Energy-Aware Data Delivery Framework for Safety-Oriented Mobile IoT,” IEEE Sensors Journal, vol. 17, no. 19, pp. 6463-6473, October 2017. Article (CrossRef Link)

[9] J. Chudzikiewicz, J. Furtak, and Z. Zielinski, "Fault-tolerant techniques for the Internet of Military Things," in Proc. of IEEE 2nd World Forum on Internet of Things (WF-IoT), pp. 496-501, December 2015. Article (CrossRef Link)

[10] Y. Peng, X. Wang, L. Guo, Y. Wang, and Q. Deng, "An Efficient Network Coding-Based Fault-Tolerant Mechanism in WBAN for Smart Healthcare Monitoring Systems," MDPI, Applied Sciences, vol. 7, no. 8, 817, 2017. Article (CrossRef Link)

[11] G. Abdul-Salaam, A. H. Abdullah, and M. H. Anisi "Energy-Efficient Data Reporting for Navigation in Position-Free Hybrid Wireless Sensor Networks," IEEE Sensors Journal, vol. 17, iss. 7, April 2017. Article (CrossRef Link)

[12] A. S. Rostami, M. Badkoobe, F. Mohanna, et. al., "Survey on clustering in heterogeneous and homogeneous wireless sensor networks," The Journal of Supercomputing, vol. 74, iss. 1, pp. 277-323, January 2018. Article (CrossRef Link)

[13] A. Mouapi and N. Hakem, "A New Approach to Design Autonomous Wireless Sensor Node Based on RF Energy Harvesting System,” MDPI, Sensors, vol. 18, no. 1, 133, January 2018. Article (CrossRef Link)

[14] I. Shin, M. Kim, and H. Choo, "On Generating Backbone Trees through Robust Multi-hop Clusters in Wireless Sensor Networks," in Proc. of Asia-Pacific Conference on Computer-Human Interaction (APCHI), LNCS 5068, pp. 30-39, July 2008. Article (CrossRef Link) 
[15] I. Shin, M. Kim, M. W. Mutka, H. Choo, and T.-J. Lee, "MCBT: Multi-hop Cluster Based Stable Backbone Trees for Data Collection and Dissemination in WSNs,” MDPI, Sensors, vol. 9, no. 8, pp. 6028-6045, July 2009. Article (CrossRef Link)

[16] T. Rault, A. Bouabdallah, and Y. Challal, "Energy efficiency in wireless sensor networks: A top-down survey,” Elsevier, Computer Networks, vol. 67, pp. 104-122, July 2014. Article (CrossRef Link)

[17] M. Kim, E. Jeong, Y.-C. Bang, S. Hwang, C., and B. Kim, "Multipath Energy-Aware Routing Protocol in Wireless Sensor Networks," in Proc. of IEEE International Conference on Networked Sensing Systems, pp. 127-130, June 2008. Article (CrossRef Link)

[18] M. Kim, E. Jeong, Y.-C. Bang, S. Hwang, C. Shin, G.-J. Jin, and B. Kim, “An Energy-aware Multipath Routing Algorithm in Wireless Sensor Networks,” IEICE Transactions on Information and Systems, vol. E91-D, no. 10, pp. 2419-2427, October 2008. Article (CrossRef Link)

[19] J. Kulik, W. Heinzelman, and H. Balakrishnan, "Negotiation-Based Protocols for Disseminating Information in Wireless Sensor Networks,” Wireless Networks, vol. 8, iss. 2-3, pp. 169-185, March 2002. Article (CrossRef Link)

[20] M. Elappila, S. Chinara, and D. R. Parhi, "Survivable Path Routing in WSN for IoT applications," Elsevier, Pervasive and Mobile Computing, vol. 43, pp. 49-63, January 2018. Article (CrossRef Link)

[21] M. Kim, M. W. Mutka, S.-H. Cho, and H. Choo, "A Dissemination Protocol to Guarantee Data Accessibility within N-hops for Wireless Sensor Networks," in Proc. of IEEE Hawaii International Conference on System Sciences (HICSS-42), pp. 1-8, January 2009. Article (CrossRef Link)

[22] M. Bhardwaj, T. Garnett, and A. P. Chandrakasan, "Upper Bound on the Lifetime of Sensor Network,” in Proc. of IEEE International Conference on Communications (ICC), June 2001. Article (CrossRef Link)

[23] G. Khanna, S. Bagchi, and Y.-S. Wu, "Fault Tolerant Energy Aware Data Dissemination Protocol in Sensor Networks,” IEEE Dependable Systems and Networks (DSN), pp. 739-748, June 2004. Article (CrossRef Link)

[24] R. Khosla, X. Zhong, G. Khanna, S. Bagchi, and E. J. Coyle, "Performance Comparison of SPIN based Push-Pull Protocols," in Proc. of IEEE Wireless Communications and Networking Conference (WCNC), pp. 3993-3998, March 2007. Article (CrossRef Link)

[25] M. H. Anisi, G. Abdul-Salaam, M. Y. I. Idris, A. W. A. Wahab, and I. Ahmedy, "Energy harvesting and battery power based routing in wireless sensor networks," Springer, Wireless Networks, vol. 23, iss. 1, pp. 249-266, January 2017. Article (CrossRef Link)

[26] S. Park, M. Kim, E. Jeong, and Y.-C. Bang, "RESS: A Data Dissemination Protocol Using Residual Energy and Signal Strength for Wireless Sensor Networks,” in Proc. of International Conference on Ubiquitous Intelligence and Computing (UIC), LNCS 6404, pp. 205-214, October 2010. Article (CrossRef Link)

[27] W. R. Heinzelman, J. Kulik, and H. Balakrishnan, "Adaptive protocols for information dissemination in wireless sensor networks," in Proc. of ACM/IEEE International Conference on Mobile Computing and Networking (MobiCom), pp. 174-185, August 1999. Article (CrossRef Link)

[28] V. B. Krishna, “An Energy-Efficient P2P Protocol for Validating Measurements in Wireless Sensor Networks,” Master Thesis, Electrical and Computer Engineering, University of Illinois at Urbana-Champaign, 2016. Article (CrossRef Link)

[29] V. B. Krishna, M. Rausch, B. E. Ujcich, I. Gupta, and W. H. Sanders, "REMAX: Reachability-Maximizing P2P Detection of Erroneous Readings in Wireless Sensor Networks,” in Proc. of IEEE/IFIP International Conference on Dependable Systems and Networks (DSN), pp. 321-332, June 2017. Article (CrossRef Link)

[30] J. Seo, M. Kim, S.-H. Cho, and H. Choo “An Energy and Distance Aware Data Dissemination Protocol Based on SPIN in Wireless Sensor Networks,” in Proc. of International Conference on Computational Science and its Applications (ICCSA), LNCS 5072, pp. 928-937, June 2008. Article (CrossRef Link) 
[31] J. Seo, M. Kim, H. Choo, and M. W. Mutka, "EDAS: Energy and Distance Aware Protocol Based on SPIN for Wireless Sensor Networks,” LNCS Transactions on Computational Science VI, vol. 5730, pp. 115-130, December 2009. Article (CrossRef Link)

[32] Scalable Network Technologies web site, Article (CrossRef Link)

[33] A. Zarrad and I. Alsmadi, "Evaluating network test scenarios for network simulators systems," International Journal of Distributed Sensor Networks, vol. 13, no. 10, October 2017. Article (CrossRef Link)

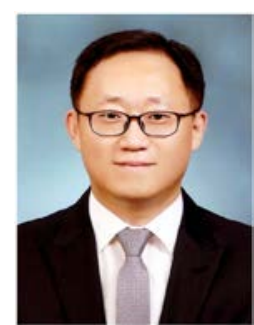

Moonseong Kim received the M.S. degree in Mathematics, August 2002 and the Ph.D. degree in Electrical and Computer Engineering, February 2007 both from Sungkyunkwan University, Korea. He was a Research Professor at Sungkyunkwan University in 2007. From December 2007 to October 2009, he was a Research Associate in ECE and CSE, Michigan State University, USA. He was a Deputy Director and a Patent Examiner with Korean Intellectual Property Office, Daejeon, Korea, from October 2009 to August 2018. In September 2018, he joined the Department of Liberal Arts, Seoul Theological University, Bucheon, Korea, where he is currently working as an Assistant Professor. His research interests include wired/wireless networking, sensor networking, mobile computing, network security protocols, and simulations/numerical analysis. Since March 2009, he has been an editor of KSII Transactions on Internet and Information Systems (TIIS).

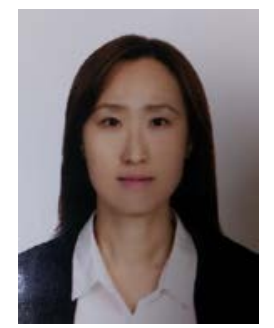

Sooyeon Park received the B.S., M.S., and Ph.D. degrees in Computer Engineering from Korea Polytechnic University, Korea, in 2004, 2007, and 2011, respectively. From 2011 to 2015, she was a part-time researcher with Korea Electronics Technology Institute, Korea. Since 2017, she has been a researcher in Computational Electromagnetics Group, Department of Electrical Engineering, Incheon National University, Incheon, Korea. Her research interests include wired/wireless networking, sensor networking, internet of things, and network security protocols.

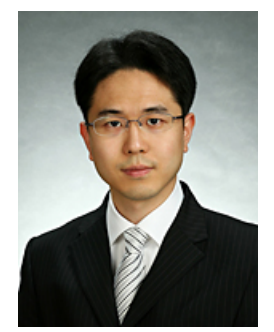

Woochan Lee received the B.S. and M.S. degrees in electrical engineering from Seoul National University, Seoul, Korea, in 2002 and 2005, respectively, and the Ph.D. degree in electrical and computer engineering from Purdue University, West Lafayette, IN, USA, in 2016. He was commissioned as a Full-time Lecturer and a First Lieutenant with the Korea Military Academy, Seoul, Korea, from 2005 to 2008. He was a Deputy Director and a Patent Examiner with Korean Intellectual Property Office, Daejeon, Korea, from 2004 to 2017. In 2017, he joined the Department of Electrical Engineering, Incheon National University, Incheon, Korea, where he is currently working as an Assistant Professor. His current research interests include computational electromagnetics, numerical analysis, and IoT applications with machine learning. 